\title{
Comparison of clock drawing with Mini Mental State Examination as a screening test in elderly acute hospital admissions
}

\author{
John Death, Adam Douglas ${ }^{1}$ and Rose Anne Kenny
}

Department of Geriatric Medicine/Medicine, Royal Victoria Infirmary, Newcastle upon Tyne NE1 4LP and ${ }^{1}$ Newcastle University Medical School, Newcastle, UK

\begin{abstract}
Summary: Clock drawing is a quick, easy to remember test that is well received by patients. It is a good screening test for Alzheimer's disease in the outpatient setting. We evaluated its usefulness compared with the standard Mini Mental State Examination (MMSE) in elderly acute medical and surgical hospital admissions. Within 48 hours of admission, 117 patients over 70 years old were administered the MMSE and asked to draw a clock. Using the MMSE as the standard, clock drawing had a sensitivity of $77 \%$ and a specificity of $87 \%$. Patients with discrepant scores were then further evaluated. The findings suggest that normal clock drawing ability reasonably excludes cognitive impairment or other causes of an abnormal MMSE in elderly acute medical and surgical hospital admissions, where cognitive impairment is common and frequently missed.
\end{abstract}

\section{Introduction}

Cognitive impairment is found in up to $30 \%$ of general medical patients and between 30 and $75 \%$ of these are unrecognized by the attending doctors. ${ }^{1}$ Unsuspected cognitive impairment causes diagnostic difficulties when the history is inaccurate and therapeutic difficulties when instructions are given with which the patient cannot comply. ${ }^{2}$

Initially used to detect temporo-parietal dysfunction, especially in stroke, clock drawing has been shown to correlate well with a diagnosis of Alzheimer's disease in outpatients. ${ }^{3,4} \mathrm{We}$ have used clock drawing in conjunction with the Mini Mental State Examination (MMSE), ${ }^{5}$ a well-validated measure of cognitive function, to screen patients in the geriatric rehabilitation unit and were impressed by the correlation between the two. Clock drawing had the added benefits of being easier to administer to the hearing impaired and more acceptable to the very ill. Patients who refuse to answer MMSE questions because they are insulted, uncertain, or both, find clock drawing less threatening. For house officers it is quick and easy to remember. Because of the high proportion of elderly admissions to hospital with cognitive impairment and the large numbers who remain undetected, a simple, sensitive screening test is required.

We therefore evaluated clock drawing as a screening test for cognitive impairment or other

Correspondence: Rose Anne Kenny, M.D., F.R.C.P.I. Accepted: 12 May 1993 causes of an abnormal Mini Mental State Examination in consecutive elderly patients early in their admission to medical and surgical wardso

\section{Method}

Consecutive patients over 70 years of age admitted to three general hospitals in Newcastle, including all surgical and medical wards, were examined within 48 hours of their admission. Each was given a piece of paper with a $10 \mathrm{~cm}$ heavy black circle with a dot in the centre printed on it. They were asked 'Imagine this is a clock face. Please fill in the numbers on the clock face.' This was repeated if asked but no other instructions were given, even if requested. If a patient spontaneously recognized an error and requested to correct it, he or she was invited to do so. An MMSE was then performed.

The MMSE is a sequence of 19 questions and tasks which evaluates orientation, immediate and short-term recall, concentration, language and construction skills. It is scored out of 30 possible points. $^{5}$

Patients who were unable to write at all were excluded. Patients judged by the ward sister to be too ill and those with prohibitive hearing and/or visual defects were also excluded. Two patients did not give consent. Clocks were classified as detailed below. Classifications were: bizarre (class 1), major spacing abnormality (class 2), minor spacing abnormality or single missing or extra number 
(class 3 ), and completely normal (class 4 ). Clocks were scored by a different author blind to the MMSE score. Clocks class 1 and 2 indicate cognitive impairment and class 3 and 4 no cognitive impairment. ${ }^{3}$ MMSE scores less than or equal to 23 were taken to indicate cognitive impairment and 24 and above to indicate no cognitive impairment. ${ }^{5-7}$ When a discrepancy occurred the patient was reviewed according to DSM III diagnostic criteria for delirium and dementia by examining case notes and psychological and, where appropriate, physical examination. ${ }^{8}$

Cognitive impairment and normality according to clock and MMSE score were then compared and sensitivity, specificity, and positive and negative predictive values calculated.

\section{Classification of clock drawing}

\section{Class 1-bizarre clocks}

These range from a few uninterpretable squiggles to perseverative use of numbers all around the perimeter. Some patients could not apply themselves well enough to make any mark at all (Examples 1-4, Figure 1).

\section{Class 2-major spacing abnormality}

These clocks contain all the correct numbers but are spaced very poorly. All 12 numbers may be bunched around one side of the clock and if the bunching finishes with the 11 (or 12 if started with 1) before the 9 o'clock horizontal, a major spacing abnormality is present (Example 5, Figure 1). There may be several bunches of numbers (Example 6, Figure 1). One number may be omitted or an extra number (usually a second 12 ) may be added. Multiple number omissions or additions belong to Class 1.

\section{Class 3-minor spacing abnormality}

The numbers are correct but spacing is slightly abnormal. If the numbers finish above the 9 o'clock horizontal it is included here (Example 7, Figure 1). When a more major spacing abnormality is recognized spontaneously (usually at the 3 or 6 location) and corrected normally for the remainder of the clock, or when there are single number errors with normal spacing, they are included here.

\section{Class 4-normal clocks}

These are completely normal. Filling in 12, 3, 6 and 9 only in the correct location is classed as normal, as is using minutes $(5,10,15,20$, etc. $)$ in the correct location.

It is very difficult to space the numbers correctly if the piece of paper is rotated so that the base of the numbers face the centre. If the subject attempted this he or she was asked to start again and not to turn the paper. If subjects spontaneously recognized a spacing abnormality they were invited to correct it if they wanted to. Where numbers were drawn away from the perimeter of the circle, they were judged according to the spacing of the numbers themselves.

\section{Results}

A total of 117 patients were studied: 69 women, 48 men, mean age 79 years (range 70-94 years). Eleven were excluded from study. Eighty-two scored 24 and over on MMSE and 35 scored 23 or below. Seventy-nine drew clocks 3 or 4 and 38 drew cloks 1 or 2 (Figure 1). Eleven patients scored 24 or above on MMSE and drew clocks class 1 or 2, while eight scored 23 or below on MMSE and drew clocks class 3 or 4 (Table I). The ability of a normal clock (class 3 or 4 ) to predict an MMSE score of 24 or above (the negative predictive value) was $90 \%$. The ability of an abnormal clock (class 1 or 2) to predict an MMSE score 23 or below (positive predictive value) was $71 \%$.

Details of the patients with discrepant results are found in Table II. Of the 11 who had 'normal' MMSE scores and drew abnormal clocks, eight had normal mental states (DSM III diagnostic criteria), two were uncertain and one had probable dementia as part of a multi-infarct state. Of the eight who had 'abnormal' MMSE scores and drew normal clocks, four had normal mental states, two had stroke-related dementia and two had Alzheimer's type dementia, one of whom had delirium as well.

\section{Discussion}

Cognitive impairment is found in over one third of elderly general medical patients. ${ }^{1}$ Its diagnosis is frequently overlooked both in the community and in acute hospital admissions, resulting in diagnostic and therapeutic difficulties. ${ }^{2}$ A simple and effective screening test to identify underlying impairment, but which can be easily carried out and interpreted by busy surgical and medical junior doctors, is required. The present study highlights clock drawing as such a sensitive and specific test which can be performed quickly.

Clock drawing requires visuospatial orientation, concentration and planning ability. The latter two 


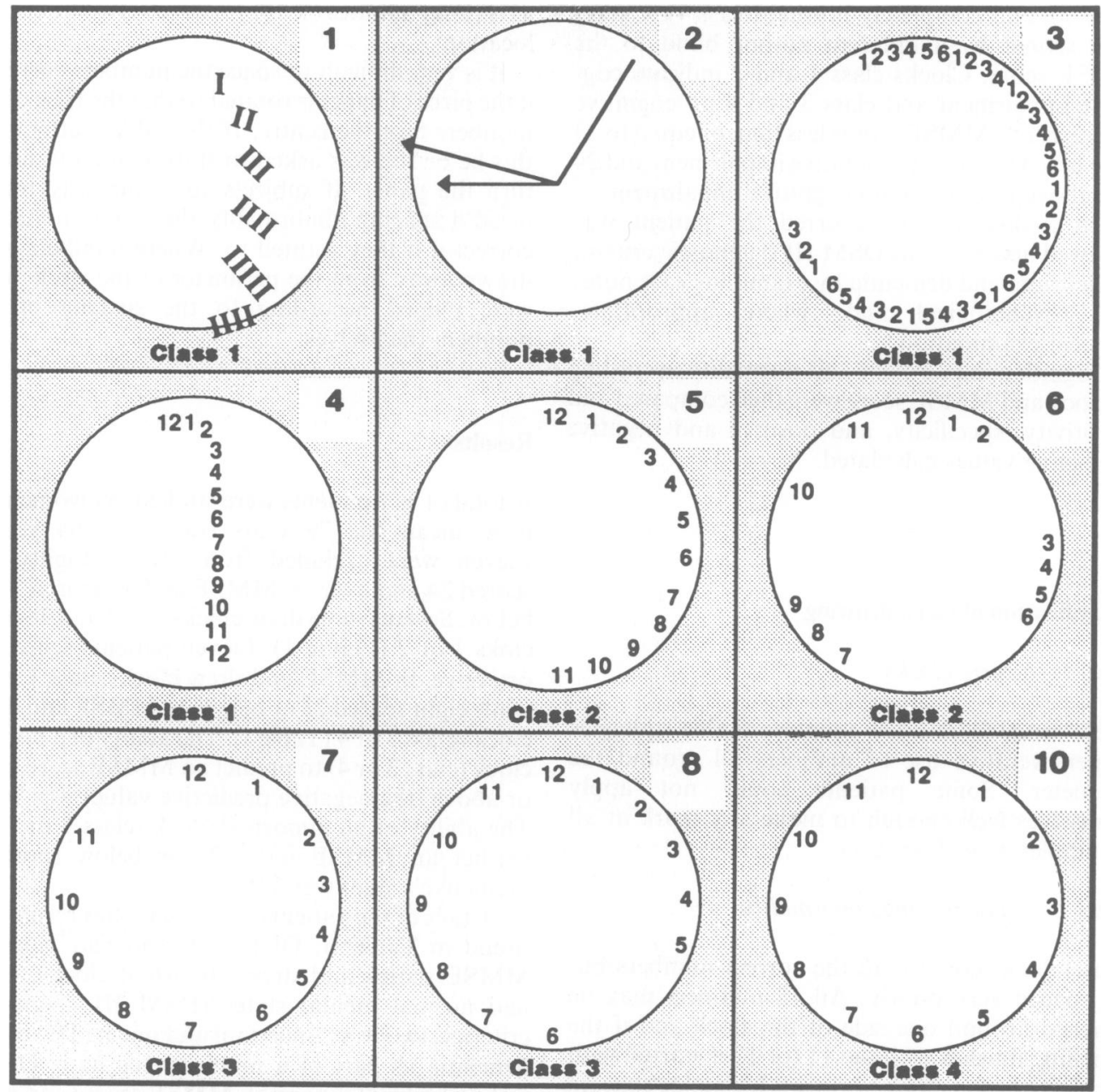

Figure 1 Classification of clocks - class 1 to 4 .

Table I Classification of clocks for MMSE scores of less than 23 or greater than 24

\begin{tabular}{lccc}
\hline \multicolumn{4}{c}{ MMSE score } \\
$\begin{array}{l}\text { Clock } \\
\text { score }\end{array}$ & $\leqslant 23$ & $\geqslant 24$ & Total \\
\hline 1 or 2 & 27 & 11 & 38 \\
3 or 4 & 8 & 71 & 79 \\
Total & 35 & 82 & 117 \\
Sensitivity & & $=77 \%$ & \\
Specificity & & $=87 \%$ & \\
Positive predictive value & $=71 \%$ \\
Negative predictive value & $=90 \%$ \\
\hline
\end{tabular}

are just as important in the ability of the test to detect the cognitively impaired as the former. If an elderly patient draws a normal clock or one with minor spacing abnormalities, the patient has a good chance of being cognitively normal. Finding 'normal' clock drawing ability in elderly, recently admitted patients reasonably excludes significant cognitive impairment. It is a test that is easy to use and easy to remember. Finding 'abnormal' clock drawing ability necessitates further assessment of cognition and affect. The difficult classification of $D$ many of the clearly abnormal clocks ${ }^{3,4}$ is not necessary. A minor spacing abnormality or a N spontaneously corrected spacing abnormality is all that needs to be recognized.

The method described is easy for the visually $\underset{\omega}{N}$ impaired to follow: a large thick circle rather than their own hand writing. The instruction used by 0 Wolff-Klein et al. ${ }^{3}$ was modified because quite reasonable questions are generated by just instructing the patient to 'draw a clock'. Still there were 11 cognitively normal patients without evidence of cerebral insult who could not do this seemingly 
Table II Evaluation of discrepant results

\begin{tabular}{|c|c|c|c|c|}
\hline$M M S E$ & Clock & Age & $\operatorname{Sex}$ & Final evaluation of mental state \\
\hline \multicolumn{5}{|c|}{$M M S E \geqslant 24:$ clock 1 or 2} \\
\hline 29 & 2 & 85 & $\mathbf{F}$ & Normal \\
\hline 27 & 1 & 82 & $\mathbf{F}$ & Normal \\
\hline 26 & 1 & 94 & $\mathbf{F}$ & Uncertain \\
\hline 26 & 1 & 84 & $\mathbf{F}$ & Normal \\
\hline 26 & 2 & 81 & $\mathrm{~F}$ & Normal \\
\hline 26 & 2 & 88 & $\mathbf{F}$ & Uncertain \\
\hline 26 & 1 & 73 & $\mathbf{F}$ & Normal \\
\hline 25 & 1 & 78 & $\mathbf{M}$ & Abnormal (multi-infarct state) \\
\hline 24 & 2 & 78 & $\mathbf{F}$ & Normal \\
\hline 24 & 2 & 75 & $\mathbf{F}$ & Normal \\
\hline 24 & 1 & 81 & F & Normal (stroke with constructional apraxia) \\
\hline \multicolumn{5}{|c|}{$M M S E \leqslant 23:$ clock 3 or 4} \\
\hline 22 & 3 & 79 & $\mathbf{M}$ & Normal \\
\hline 22 & 3 & 78 & $\mathbf{F}$ & Normal (stroke) \\
\hline 21 & 3 & 73 & $\mathbf{F}$ & Abnormal (multi-infarct state) \\
\hline 19 & 3 & 78 & $\mathbf{M}$ & Abnormal (mild Alzheimer's dementia + delirium) \\
\hline 18 & 3 & 75 & $\mathbf{M}$ & Abnormal (multi-infarct dementia) \\
\hline 15 & 4 & 82 & $\mathbf{M}$ & Normal (chronic pain, carcinoma of the rectum) \\
\hline 13 & 3 & 87 & $\mathbf{F}$ & Abnormal (Alzheimer's disease) \\
\hline 12 & 4 & 80 & $\mathbf{M}$ & Normal (carcinoma of the lung, exhaustion) \\
\hline
\end{tabular}

simple task and the explanation for this is unclear.

The Mini Mental State Examination for Cognitive Function is widely used and has been extensively evaluated. ${ }^{5-7}$ Its best documented short fall relates to false-positive results (that is, low scores) in cognitively normal elderly subjects with minimal education. ${ }^{7-9}$ False-negative results are less common and this was the important consideration from our study. We did not attempt to identify causes of cognitive impairment in this study. When used as a screening test for acute admissions, the cause of cognitive impairment is not so important; only whether it is present is important. ${ }^{13}$ Further evaluation can wait. ${ }^{3}$ Choice of a cut-off point for dementia using the MMSE is complex and predictive values for the test vary according to the population studied ${ }^{10,14}$ and educational level of the

\section{References}

1. McLean, S. Assessing dementia. Difficulties, definitions and differential diagnosis. Aust NZ J Psychiatry 1987, 21: 142-174.

2. Black, D.A. Mental state and presentation of myocardial infarction in the elderly. Age Ageing 1987, 16: 125-127.

3. Wolff-Klein, G.P., Silverstone, F.A., Levy, A.P. \& Brod, M.S. Screening for Alzheimer's disease by clock drawing. $J$ Am Geriatr Soc 1989, 37: 730-734.

4. Sunderland, T., Lawlor, B.A., Molchan, S.E. \& Martinez, R.A. Clock drawing in Alzheimer's disease. J Am Geriatr Soc 1989, 37: 725-729.

5. Folstein, M., Folstein, S. \& McHugh, P. Mini Mental State. A practical method for grading the cognitive state of patients for the clinician. J Psychiatr Res 1975, 12: 189-198. subjects. ${ }^{9,11,12}$ When compared with the MMSE, an abnormal clock drawing test can alert the admitting doctor to the necessity for further testing. Rather than excluding cognitive impairment, the test is proposed as a simple and rapid tool for use by admitting junior staff to highlight possible cognitive impairment or other causes of abnormal MMSE testing, such as affective disorders and delirium; diagnoses which are frequently overlooked in such patients and can significantly influence patient management and response to treatment. ${ }^{9,13}$

Clock drawing is a practical and sensitive screening test for routine assessment of the elderly in general medical and surgical wards where up to a third of elderly patients can be expected to have some degree of cognitive impairment.
6. Anthony, J.C., Le Reschel, Niaz, U., von Korff, M.R. \& Folstein, M.F. Limits of the mini mental state as a screening test for dementia among hospital patients. Psychol Med 1982, 12: $397-408$

7. Folstein, M., Anthony, J.C., Parhad, I., Duffy, B. \& Gruenberg, E.M. The meaning of cognitive impairment in the elderly. J Am Geriatr Soc 1985, 33: 228-235.

8. American Psychiatric Association, Committee on Nomenclature and Statistics. Diagnostic and Statistical Manual of Mental Disorders, 3rd ed. American Psychiatric Association, Washington, DC, 1987, pp. 100-103.

9. Ainslie, N.K. \& Murden, R.A. Effect of education on the clock-drawing dementia screen in non-demented elderly persons. J Am Geriatr Soc 1993, 41: 249-252. 
10. O'Connor, D.W., Pollitt, P.D., Hyde, J.B. et al. The reliability and validity of the Mini Mental State Examination in a British community. J Psychiatr Res 1989, 23: 87-96.

11. Pfeiffer, E. A short portable mental state questionnaire for the assessment of organic brain deficit in elderly patients. $\mathrm{J} \mathrm{Am}$ Geriatr Soc 1975, 23: 433-441.

12. Murden, R.A., McRae, T.D., Kawer, S. et al. Mini Mental State Exam scores vary with education in blacks and whites. $J$ Am Geriatr Soc 1991, 39: 149-155.
13. Levkoff, S.E., Evans, D.A., Liptzin, B. et al. Delirium. The occurrence and persistence of symptoms among elderly hospitalized patients. Arch Intern Med 1992, 152: 334-340.

14. Siu, A.L. Screening for dementia and investigating its causes. Ann Intern Med 1991, 115: 122-132. 